\title{
A REGULARITY THEOREM FOR THE FIRST VARIATION OF THE AREA INTEGRAND ${ }^{1}$
}

\author{
BY WILLIAM K. ALLARD
}

Communicated by J. J. Kohn, April 8, 1971

Suppose $R^{n}$ is $n$-dimensional Euclidean space, $k$ is an integer and $2 \leqq k<n$. We will state here a regularity theorem for $k$-dimensional varifolds in $R^{n}$ which satisfy inequalities involving the first variation of their weighted $k$-dimensional area. Naturally formulated analogues of our results hold in any Riemannian manifold.

Though quite simple, the concept of a varifold is unfamiliar to the general mathematical public. In order to suggest the nature of our results in a context familiar to geometers, we first state two corollaries to our regularity theorem in which the varifolds under consideration are submanifolds of $R^{n}$. Let $M_{k}\left(R^{n}\right)$ be the set of continuously differentiable $k$-dimensional submanifolds of $R^{n}$ of locally finite $k$ dimensional area; whenever $M \in M_{k}\left(R^{n}\right)$, let $\|M\|$ be the Radon measure on $R^{n}$ which assigns to each open subset of $R^{n}$ the $k$-dimensional area of its intersection with $M$. Whenever $a \in R^{n}$ and $0<r<\infty$, let $U(a, r)=R^{n} \cap\{x:|x-a|<r\}$ and let $S^{n-1}$ be the boundary of $U(0,1)$. Let $\alpha(k)$ be the $k$-dimensional area of the ball of radius 1 centered at 0 in $R^{k}$.

COROllary. Corresponding to each $p$ with $k<p<\infty$ and each $\epsilon$ with $0<\epsilon<1$ there is $\eta>0$ with the following property:

Suppose

(a) $M \in M_{k}\left(R^{n}\right)$ and $0 \in M$;

(b) $\|M\| U(0,1) \leqq(1+\eta) \alpha(k)$;

(c) $M$ is smooth, $M$ is closed relative to $U(0,1)$ and

$$
k\left(\int_{U(0,1)}|H(x)|^{p} d\|M\|_{x}\right)^{1 / p} \leqq \eta,
$$

where $H$ is the mean curvature vector of $M$.

Then there are a linear isometry $\theta: R^{n} \rightarrow R^{n}$ and smooth functions $f_{j}: R^{k} \rightarrow R, j=1, \cdots, n-k$, such that

AMS 1970 subject classifications. Primary 49F22; Secondary 49F10, 53A10.

Key words and phrases. First variation of area integrand, regularity of weak solutions to variational inequalities, manifold, varifold, mean curvature.

1 This work was supported in part by the National Science Foundation. The author is a Fellow of the Alfred P. Sloan Foundation.

Copyright (c) American Mathematical Society 1971 


$$
\begin{array}{r}
U(0,1-\epsilon) \cap \theta(M)=U(0,1-\epsilon) \cap\left\{x: x_{k+j}=f_{j}\left(x_{1}, \cdots, x_{k}\right),\right. \\
j=1, \cdots, n-k\}
\end{array}
$$

and such that

$$
\left|D_{i} f_{j}(y)-D_{i} f_{j}(z)\right| \leqq \epsilon|y-z|^{1-k / p}
$$

whenever $y, z \in R^{k}$ and $i=1, \cdots, k, j=1, \cdots, n-k$.

Our next corollary is a rigidity theorem.

COROLLARY. There is a positive number $\eta$ with the following property:

Suppose $L$ is a smooth closed $(k-1)$-dimensional submanifold of $S^{n-1}, L$ has zero mean curvature relative to $\mathbf{S}^{n-1}$ and the $(k-1)$-dimensional area of $L$ does not exceed $(1+\eta) k \alpha(k)$. Then there is a $k$-dimensional linear subspace $T$ of $R^{n}$ such that $L=T \cap S^{n-1}$.

We assert that the conclusions of the first corollary hold when (a), (b), (c) are naturally reformulated to apply to a class of objects far more general than the submanifolds of $R^{n}$. This reformulation will imply a statement which includes the second corollary. Regularity theorems have been obtained for flat chain solutions to minimum problems defined by parametric elliptic integrands; see [2]. Although we study here only the area integrand, which is the simplest parametric integrand, we assume in no way that the surfaces considered minimize area; we do not even assume that the first variation of their area is zero, only that this first variation is summable to a power greater than the dimension of the surface.

Following Almgren (see [1]), we define a $k$-dimensional varifold in $R^{n}$ to be a Radon measure on $R^{n} \times G(n, k)$ where $G(n, k)$ is the Grassmann manifold of $k$-dimensional linear subspaces of $R^{n}$. Let $V_{k}\left(R^{n}\right)$ be the weakly topologized space of these varifolds. When $V \in V_{k}\left(R^{n}\right)$, let $\|V\|(A)=V(A \times G(n, k))$ for $A \subset R^{n} ;\|V\|$ is called the weight of $V$. Whenever $M \in M_{k}\left(R^{n}\right)$, let

$$
\boldsymbol{v}(M)(B)=\|M\|\{x:(x, \operatorname{Tan}(M, x)) \in B\}, \quad B \subset R^{n} \times G(n, k),
$$

where $\operatorname{Tan}(M, x) \in G(n, k)$ is the tangent space to $M$ at $x \in M$; clearly, $v(M) \in V_{k}\left(R^{n}\right)$ and $\|v(M)\|=\|M\|$. Given $M_{1}, M_{2}, \cdots$ $\in M_{k}\left(R^{n}\right)$ positive real numbers $c_{1}, c_{2}, \cdots$ and a Borel subset $B$ of $\boldsymbol{R}^{n}$ such that $\sum_{i=1}^{\infty} c_{i}\left\|M_{i}\right\|(B \cap K)<\infty$ for every compact subset $K$ of $\boldsymbol{R}^{n}$, we see that the restriction of $\sum_{i=1}^{\infty} c_{i} v\left(M_{i}\right)$ to $B \times G(n, k)$ is a member of $V_{k}\left(R^{n}\right)$. Such a varifold is called rectifiable; if the $c_{i}$ may be taken to be positive integers, it is called integral. The class of integral varifolds includes all classical geometric objects such as real 
analytic sets and continuously differentiable curvilinear complexes, as well as objects of more general singularity structure.

Suppose $F: R^{n} \rightarrow R^{n}$ is a univalent continuously differentiable mapping such that at each point $x \in R^{n}$ the differential $D F(x)$ of $F$ at $x$ is nonsingular. For each $M \in M_{k}\left(R^{n}\right)$, let $F_{\sharp} M=\{F(x): x \in M\}$ $\in M_{k}\left(R^{n}\right)$. We now extend $F_{\#}$ to $V_{k}\left(R^{n}\right)$. At each $(x, S) \in R^{n} \times G(n, k)$, let $J_{k} F(x, S)$ be the $k$-dimensional area of $D F(x)(S \cap U(0,1))$ divided by $\boldsymbol{\alpha}(k)$; for each $V \in V_{k}\left(R^{n}\right)$, let $F_{\sharp} V \in V_{k}\left(R^{n}\right)$ be such that

$$
F_{\# V(B)}=\int_{\{(x, S):(F(x), D F(x)(S)) \in B\}} J_{k} F(x, S) d V(x, S)
$$

for each Borel subset $B$ of $R^{n} \times G(n, k)$. It is then elementary that the diagram

$$
\begin{array}{ll}
V_{k}\left(R^{n}\right) \stackrel{F_{\sharp}}{\longrightarrow} V_{k}\left(R^{n}\right) \\
v \uparrow & \quad v \uparrow \\
M_{k}\left(R^{n}\right) \stackrel{F_{\sharp}}{\longrightarrow} M_{k}\left(R^{n}\right)
\end{array}
$$

is commutative. Now let $\mathfrak{X}\left(R^{n}\right)$ be the vector space of smooth functions $g: R^{n} \rightarrow R^{n}$ with compact support. For each $V \in V_{k}\left(R^{n}\right)$ we let

$$
\delta V: \mathfrak{X}\left(R^{n}\right) \rightarrow R
$$

be the linear functional which has at $g \in \mathfrak{X}\left(R^{n}\right)$ the value

$$
d /\left.d t\left\|h_{t \sharp} V\right\|(U)\right|_{t=0},
$$

where $h_{t}(x)=x+\operatorname{tg}(x)$ for $(t, x) \in R \times R^{n}$ and $U$ is a bounded open subset of $R^{n}$ containing the support of $g$. As is well known,

$$
\delta v(M)(g)=-k \int g(x) \cdot H(x) d\|M\| x
$$

whenever $M \in M_{k}\left(R^{n}\right), M$ is smooth, $H$ is the mean curvature vector of $M, g \in \mathfrak{X}\left(\boldsymbol{R}^{n}\right)$ and the support of $g$ meets $M$ in a closed set. Thus $\delta V$ is in some sense the mean curvature of $V$.

Whenever $V \in V_{k}\left(R^{n}\right), 1<p<\infty$, and $G$ is an open subset of $R^{n}$, we let $\boldsymbol{v}(V ; p, G)$ be the supremum of the set of numbers $\delta V(g)$ corresponding to those $g \in \mathfrak{X}\left(R^{n}\right)$ such that support $g \subset G$ and $\left(\int|g(x)|^{q} d\|V\| x\right)^{1 / q} \leqq 1$, where $q=p /(p-1)$. For example, if $M \in M_{k}\left(R^{n}\right), M$ is smooth, $M$ is closed relative to $G$ and $H$ is the mean curvature vector of $M$, it is easy to see that

$$
v(v(M) ; p, G)=k\left(\int_{G}|H(x)|^{p} d\|M\| x\right)^{1 / p} .
$$


If $\delta V(g)=0$ for all $g \in \mathfrak{X}\left(\boldsymbol{R}^{n}\right)$ with support $g \subset G$, we say $V$ is stationary in $G$.

We now state our main result.

Regularity Theorem. Corresponding to each $p$ with $k<p<\infty$ and each $\epsilon$ with $0<\epsilon<1$ there is $\eta>0$ with the following property:

Suppose

(a) $V \in V_{k}\left(R^{n}\right), 0 \in$ support $\|V\|$ and

$$
\limsup _{r \rightarrow 0} \frac{\|V\| U(x, r)}{\alpha(k) r^{k}} \geqq 1 \quad \text { for }\|V\| \text { almost all } x \in U(0,1) ;
$$

(b) $\|V\| U(0,1) \leqq(1+\eta) \alpha(k)$;

(c) $v(V ; p, U(0,1)) \leqq \eta$.

Then there are a linear isometry $\theta: R^{n} \rightarrow R^{n}$ and continuously differentiable functions $f_{j}: R^{k} \rightarrow R, j=1, \cdots, n-k$, such that

$$
\begin{aligned}
& U(0,1-\epsilon) \cap \theta(\text { support }\|V\|) \\
& \quad=U(0,1-\epsilon) \cap\left\{x: x_{k+j}=f_{j}\left(x_{1}, \cdots, x_{k}\right), j=1, \cdots, n-k\right\}
\end{aligned}
$$

and such that

$$
\left|D_{i} f_{j}(y)-D_{i} f_{j}(z)\right| \leqq \epsilon|y-z|^{1-k / p}
$$

whenever $y, z \in R^{k}$ and $i=1, \cdots, k, j=1, \cdots, n-k$. Moreover,

$$
\Theta^{k}(\|V\|, x)=\lim _{r \rightarrow 0} \frac{\|V\| U(x, r)}{\alpha(k) r^{k}}
$$

is a real number for all $x \in U(0,1)$ and, for $V$ almost all $(x, S)$ $\in U(0,1-\epsilon) \times G(n, k)$,

$$
S=\operatorname{Tan}(\text { support }\|V\|, x) .
$$

The theorem is proved using geometric measure theoretic techniques, some of which appear in [1] and [3] and are known to workers in this field, together with a variant of an argument used by Almgren in [2], to exploit well-known a priori estimates for the Laplacian. To extend the theorem to Riemannian manifolds, one uses Nash's isometric imbedding theorem (see [4]) and an elementary computation involving mean curvature. This regularity theorem is the main theorem in a paper which will study other aspects of the functional $\delta V$ as well.

Suppose $V \in V_{k}\left(R^{n}\right), p$ is as in the Regularity Theorem, $G$ is an open subset of $R^{n}, \boldsymbol{v}(V ; p, G)<\infty, 0<d<\infty$, and

$$
\limsup _{\boldsymbol{r} \rightarrow \mathbf{0}}\|V\| U(x, r) / \boldsymbol{\alpha}(k) \boldsymbol{r}^{k} \geqq d
$$


for $\|V\|$ almost all $x \in G . .^{2}$ Let $S$, the singular set of $V$ in $G$, be the set of points $x$ in $G \cap$ support $\|V\|$ for which $V$ does not have near $x$ a continuously differentiable structure as in the conclusion of the Regularity Theorem. It can be shown that $S$ is closed relative to $G$ and has no interior; it is not true in general that $\|V\|(S)=0 .{ }^{3}$ Does the hypothesis that $V$ is stationary in $G$ imply that $\|V\|(S)=0$ ? The resolution of this question is an outstanding problem in the field.

Using topological arguments similar to those of Morse Theory, Almgren has proved the following theorem: Suppose $M$ is a compact Riemannian manifold and $k$ is a positive integer less than the dimension of $M$. There is a nonzero k-dimensional integral varifold which is stationary in $M$. See [1]. The regularity question for such varifolds was the main impetus for this work. Our theorem together with Morrey's work on higher differentiability (see [3]) implies that an open dense subset of the support of such a varifold is a smooth $k$-dimensional submanifold of $M$ with zero mean curvature.

In the course of this work I have benefited from conversations with H. Federer and E. Bombieri; several consultations I have had with F. J. Almgren, Jr. were indispensable, and it was at his suggestion that I began this work.

\section{REFERENCES}

1. F. J. Almgren, Jr., The theory of varifolds, Mimeographed notes, Princeton, 1965.

2. - Existence and regularity almost everywhere of solutions to elliptic variational problems among surfaces of varying topological type and singularity structure, Ann. of Math. (2) 87 (1968), 321-391. MR 37 \#837.

3. H. Federer, Geometric measure theory, Die Grundlehren der math. Wissenschaften, Band 153, Springer-Verlag, New York, 1969. MR 41 \#1976.

4. J. F. Nash, Jr., The imbedding problem for Riemannian manifolds, Ann. of Math. (2) 63 (1956), 20-63. MR 17, 782.

Princeton University, Princeton, New Jersey 08540

2 It is a theorem that the restriction of $V$ to $G \times G(n, k)$ is rectifiable.

If the oscillation of $\Theta^{k}(\|V\|, \cdot)$ on support $\|V\|$ is less than $\eta d$, it will be true that $\|v\|(S)=0$. 